\title{
Research on Non-Invasive Wireless Wearable Sensor System Using Virtual and Physical Sensors for Lower Limb Motion Analysis
}

\author{
Liu Kun ${ }^{1, \text { a }}$, Zhao Jianchen ${ }^{1, \mathrm{~b}}$, Han xuan ${ }^{1, \mathrm{c}}$ \\ ${ }^{1}$ School of Mechanical Science and Engineering, Jilin University, \\ Changchun, China

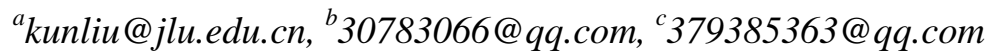

\begin{abstract}
.
Ambulatory assessment of gait posture is a promising clinical tool to diagnose walking disabilities. This paper presents a method using non-invasive wireless wearable sensor system based on virtual and physical sensors to estimate the motion of lower limb. The data measured by accelerometers and magnetometers was used to calculate the joint motion data as measured by a virtual sensor inside the joint. There is no integration of acceleration or angular velocity for the joint positions and rotations, which is an improvement on the previous methods. There are no physical sensors implanted in the knee joint based on the virtual-sensor difference based algorithm, therefore it is feasible to analyze knee joint kinematics with less numbers and types of sensors noninvasively. Finally wireless transceiver modules are integrated with the sensors and microprocessor composing a wearable sensor system to achieve real time analysis for the lower limb motion.
\end{abstract}

Keywords: Wearable Sensor System; Non-Invasive; Motion Analysis

\section{Introduction}

With the increasing of aging population, the lower limb dyskinesia of older adults caused by accidental fall becomes a big medical care problem. Therefore, the detection and analysis of lower limb motion state in the rehabilitation training are very important. Especially the ambulatory estimation of lower limb posture using handy and portable wearable sensor system is very important in the diagnosis of patients with stroke, Parkinson or knee osteoarthritis disease [1], 
and useful to evaluate the rehabilitation of patients. As well, it will be a promising clinical tool to diagnose walking disabilities. Especially the complete understanding of joint kinematics is the key point for the lower limb posture kinematic data combined with force platforms for kinetic data [2]. However, since the system is space-consuming and expensive, it is not applicable for the out-lab ambulatory estimation of lower limb posture in ordinary life. Recently, many ambulatory systems for various clinical applications have been developed to monitor physical activities, for example, to estimate body segment orientation by integrating angular velocity of the body segments measured by gyroscopes [3], or estimating knee joint $\mathrm{FE}$ angle using segmental acceleration and angular velocity data using inertial sensor-based method [4].

Therefore, in this paper, to improve on the method employed in previous literatures, we present a technique for non-invasively and quantitatively analyzing the 3D lower limb posture in the geomagnetic field using fewer kinds of sensors. This technique employs accelerometers and magnetometers, but no gyroscopes, using a simple algorithm with high accuracy suitable for Micro Control Unit (MCU), not including integration errors resulted from offsets and drifts of angular velocities, and is not restricted by the walking speed. Finally we integrated wireless transceiver modules with the sensors and MCU as handy and portable wearable sensor system to achieve real time analysis of the lower limb motion. In this paper, we present a method to analyze lower limb motion with wearable sensor system

\section{Methods and materials}

We have recently presented a double tri-axial accelerometers based approach to measure the lower limb orientation angles [5]. In the paper, only accelerometers were used to estimate the angles for lower limb orientation in 3D space. The algorithm was based on the difference between two groups of accelerations without integration, which was simple and time-saving for micro board. However, in the method, it was assumed that the lower limb segments were rigid segments and the subjects walked in a straight forward way with very little trunk sway, skin artifacts and no significant inversion/extension (i/e) rotation of the leg.

Then we presented another paper [6], an original approach based on accelerometers and magnetometers for ambulatory estimation and analysis of 3D knee joint kinematics is presented. The flexion/extension (f/e) angle, abduction/adduction (a/a) angle and inversion/extension (i/e) rotation angles in 
the anatomical joint coordinate system were estimated. A wearable sensor system composed out of inertial measurement unit composed out of an accelerometer and magnetometers was developed, and then tested on the lower limb. Without integration of angular acceleration or angular velocity for 3D lower limb joint kinematic analysis, the calculated result is not distorted by offset and drift. Another advantage of this method is that joint rotations within the anatomical coordinate system are independent of the order in which the rotations occur. This eliminates the requirement of specifying the order of the rotations, a procedure commonly believed to be necessary when Euler angles are used.

Finally, we presented a paper [7] to improve on the method employed in previous literatures. We presented a technique for visually and quantitatively analyzing the 3D lower limb posture in the geomagnetic field using fewer kinds of sensors. This technique employs accelerometers and magnetometers, but no gyroscopes, using a simple algorithm with high accuracy suitable for Micro Control Unit (MCU), not including integration errors resulted from offsets and drifts of angular velocities, and is not restricted by the walking speed. To quantitatively analyze the lower limb posture, the hip joint angles were estimated using a physical-sensor difference based algorithm, and the knee joint angles were calculated using a virtual-sensor difference based algorithm, and then the stick figures for visual confirmation of the human gait posture were shown.

As mentioned in papers [7], the main theory for the wearable sensor system is physical-sensor difference and virtual-sensor difference based method for visual and quantitative estimation of lower limb gait posture using accelerometers and magnetometers. First we estimate the hip joint f/e angle and a/a angle using physical-sensor difference based algorithm:

$$
\theta_{A A}=\sin ^{-1}\left(\left(r_{B} a_{A y}-r_{A} a_{B y}\right) /\left(\left(r_{B}-r_{A}\right) \sqrt{A_{O_{r} y}{ }^{2}+A_{O_{r} z}}\right)\right)-\sin ^{-1}\left(A_{O_{r y} y} / \sqrt{A_{O_{r} y}{ }^{2}+A_{O_{r} z}{ }^{2}}\right)
$$

$\theta_{F E}=\sin ^{-1}\left(\frac{r_{B} a_{A x}-r_{A} a_{B x}}{\left(r_{B}-r_{A}\right) \sqrt{A_{O_{r} x}{ }^{2}+A_{O_{r} y}{ }^{2}+A_{O_{r} 2}{ }^{2}-\left(\frac{r_{B} a_{A y}-r_{A} a_{B y}}{r_{B}-r_{A}}\right)}}\right)$

Then we calculate the knee joint angles using the virtual-sensor difference 
based algorithm using the following equations,

$$
\begin{aligned}
\mathbf{A}_{\mathbf{t}} & =\mathbf{R} \mathbf{A}_{\mathrm{s}} \\
\mathbf{M}_{\mathbf{t}} & =\mathbf{R} \mathbf{M}_{\mathrm{s}}
\end{aligned}
$$

where $\mathbf{R}$ is the rotation matrix between the two virtual sensors, which is also between the thigh and shank. It can be expressed with the FE angle $\varphi_{F E}$, AA angle $\varphi_{A A}$ and IE rotation angle $\varphi_{I E}$ as follow,

$$
\begin{gathered}
\mathbf{R}=\mathbf{R}_{\theta_{z}} \mathbf{R}_{\theta_{x}} \mathbf{R}_{\theta_{y}}= \\
{\left[\begin{array}{ccc}
\cos \varphi_{I E} & \sin \varphi_{I E} & 0 \\
-\sin \varphi_{I E} & \cos \varphi_{I E} & 0 \\
0 & 0 & 1
\end{array}\right]\left[\begin{array}{ccc}
1 & 0 & 0 \\
0 & \cos \varphi_{A A} & \sin \varphi_{A A} \\
0 & -\sin \varphi_{A A} & \cos \varphi_{A A}
\end{array}\right]\left[\begin{array}{ccc}
\cos \varphi_{F E} & 0 & -\sin \varphi_{F E} \\
0 & 1 & 0 \\
\sin \varphi_{F E} & 0 & \cos \varphi_{F E}
\end{array}\right]}
\end{gathered}
$$

Then the following chart in Fig.1 shows procedure of the experiment using the developed wearable sensor system. And in Fig.2 we show our new design of the external skeleton for fixing the sensors, permitting more degrees of freedom for the joint.

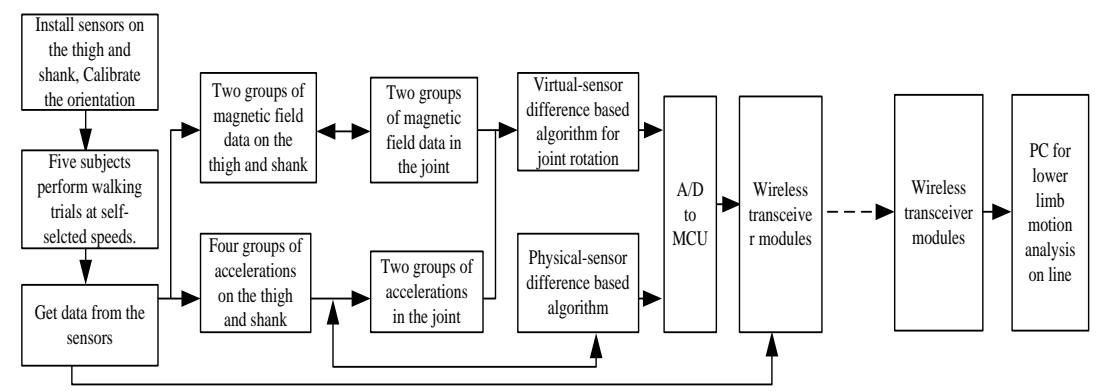

Fig.1 Procedure of the experiment using the developed wearable sensor system to estimate the lower limb motion.

\section{Experiments}

In the previous research, the external skeleton used for fixing sensors was connected with universal joint. Especially at the knee joint, a universal joint cannot allow rotations in three rotations (f/e, i/e, a/a) but just two rotations (f/e, $\mathrm{a} / \mathrm{a}$ ). To maximize the freedom of the joint allowed by the external skeleton, an improvement of the previous external skeleton joints was replaced. An assemblage of a ball socket joint and universal joint was wringed together. 
Therefore, the allowed range of the flexion/extension angle, abduction/adduction angle and inversion/extension rotation angle reached more than $60^{\circ}$.

In order to evaluate the presented method, a prototype of wearable sensor system was developed. The prototype comprised one MCU, four analog inertial sensors named $\mathrm{MAG}^{3}$ consisting of a triaxial magnetometer, an accelerometer and a gyroscope. As an improvement, we integrated two wireless transceiver modules. One was integrated on the MCU board for sending out the processed data by MCU to PC, and the other one is connected with PC for receiving data from MCU. Then the data can be used in PC for doctors to analysis the human lower limb motion and give proper diagnosis of patients or evaluate the rehabilitation of patients. As shown in Fig.3, the upside device on the left side is the MCU and the wireless transceiver modules, and the volunteer was walking wearing on the developed wearable sensor system in the working space of the optical motion system to test the system.

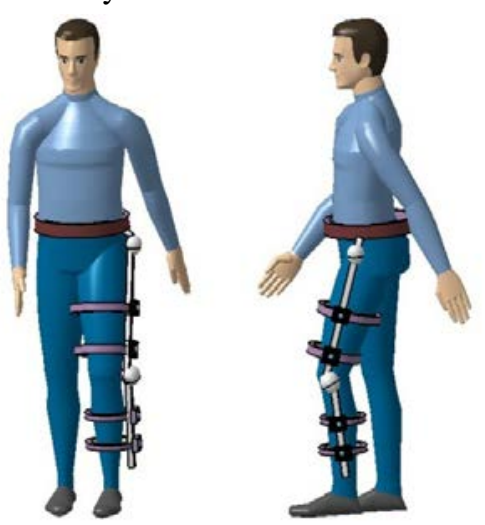

Fig.2 Design of the wearable sensor system

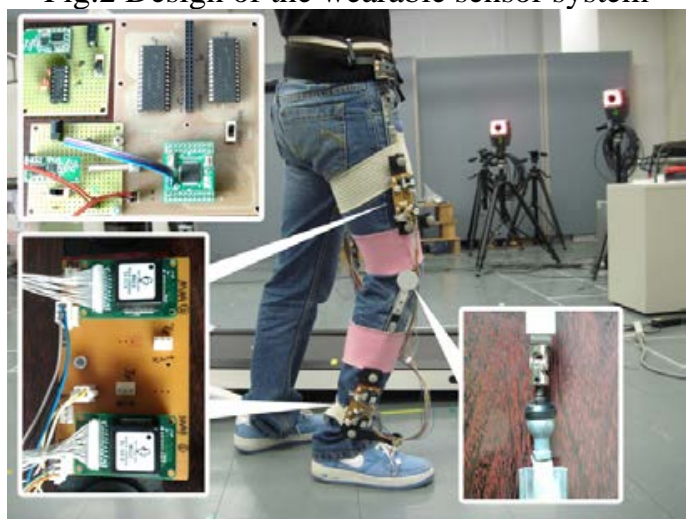

Fig.3 Experiment using the developed wearable sensor system and the external skeleton in the working space of the optical motion system. 


\section{Results}

Four groups of accelerations measured by $\mathrm{MAG}^{3} \mathrm{~s}$ were used to calculate two groups of virtual accelerations in the knee joint using the physical-sensor difference based algorithm, and then were used to calculate the knee joint angles using the virtual-sensor difference based method. Two groups of magnetic field data captured by the two $\mathrm{MAG}^{3}$ s were also used to calculate the knee joint angles using the virtual-sensor difference based method. To test the prototype in ideal conditions with less noise caused by skin motion artifact due to impact loading and muscle activation. All the readings of the sensors were recorded and processed in the MCU and sent to PC by wireless transceiver modules real time. The sampling frequency was $100 \mathrm{~Hz}$ and the A/D had a 12-bit resolution. The calculated knee joint engles were compared with the reference angles obtained from the camera system. Fig.4 shows the compared results of the FE, AA and IE angles of the knee joint in one trial.

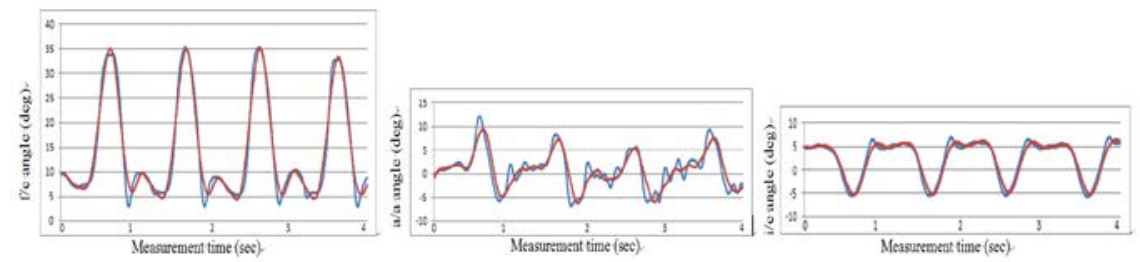

Fig.4 Two groups of the f/e angle, a/a angle and i/e rotation angle of the knee joint in the anatomical coordinate system. The red line corresponds to the developed sensor system and the blue line to the camera system.

\section{Discussion and conclusion}

From the result shown in Fig.4, the presented method showed a strong correlation with the camera system data. Our method was the first to consider the joint kinematics using accelerometers and magnetometers without gyroscopes. In this technique, gravitational acceleration, translational acceleration and noise, which were simultaneously acting on every two sensors in each sensor group, were eliminated based on the physical-sensor difference based algorithm. The virtual accelerations of the knee joint were calculated only based on the differences between the two rotational accelerations on the thigh or shank. There was no integration of angular acceleration or angular velocity for the calculation of the knee joint angles. Therefore the results were not distorted by offset and drift. Besides, it was more simple and practical to use virtual sensors than implant physical sensors in the knee joint to calculate the rotation angles. And 
we use wireless transceiver modules for sending out and receive data between the MCU and host PC real time, it made the wearable sensor system much more handy and portable for an experimenter, and it made the activity of experimenters no longer subject to detect space constraints compared with the previous wearable sensor system connected with wire. Therefore, the research for non-invasive wireless wearable sensor system for lower limb motion analysis in this paper has made a major breakthrough.

\section{Acknowledgement}

The project is supported by the National Natural Science Foundation of China (Grant No. 81101138) and China postdoctoral science foundation (Grant No. 2011M500601)

\section{References}

1. K. Turcot, R.Aissaoui, K. Boivin, M.Pelletier, N.Hagemeister, De Guise, "New accelerometric method to Discriminate between asymptomatic subjects and patients with medial knee osteoarthritis during 3-D gait," IEEE Trans. Biomed. Eng., vol. 55, no. 4, pp. 1415-1422, 2008.

2. H. Dejnabadi, B. M. Jolles, and K. Aminian, "A new approach to accurate measurement of uniaxial joint angles based on a combination of accelerometers and gyroscopes," IEEE Trans. Biomed. Eng., vol. 52, no. 8, pp. 1478-1484, 2005.

3. Dejnabadi, H., Jolles, Brigitte M., Casanova, Emilio, Fua, Pascal, Aminian, Kamiar. 2006. Estimation and visualization of sagittal kinematics of lower limbs orientation using body-fixed sensors. IEEE Transactions on Biomedical Engineering. 53 (7):1385-1392.

4. Glen Cooper, Ian Sheret, Louise McMillian, Konstantinos Siliverdis, Ning Sha, Diana Hodgins, Laurence Kenney, David Howard. 2009. Inertial sensor-based knee flexion-extension angle estimation. Journal of Biomechanics. 42 (16): 2486-2494.

5. Kun Liu, Tao Liu, Kyoko Shibata, Yoshio Inoue, Rencheng Zheng, "Novel Approach to Ambulatory Assessment of Human Segmental Orientation on a Wearable Sensor System,” J. Biomech., vol. 42, no. 16, pp. 2747-2752, 2009

6. Kun Liu, Yoshio Inoue, Kyoko Shibata, Cao Enguo. Ambulatory Estimation of Knee joint kinematics in Anatomical coordinate system Using Accelerometers and Magnetometers. IEEE Transaction on Biomedical 
Engineering. 2011, 58(2): 435-442.

7. Kun Liu, Yoshio Inoue, Kyoko Shibata. Physical-sensor difference and virtual-sensor difference based method for visual and quantitative estimation of lower limb 3D gait posture using accelerometers and magnetometers. Computer Methods in Biomechanics and Biomedical Engineering. 2012, 15(2): 203-210 